DO-TH 06/04

June 2006

\title{
Parton evolution in the fixed flavor factorization scheme
}

\author{
M. Glück and E. Reya \\ Institut für Physik, Universität Dortmund \\ D-44221 Dortmund, Germany
}

\begin{abstract}
It is argued that while the scale dependence of the parton distributions in the fixed flavor factorization scheme is governed by three active flavors, the scale dependence of the running coupling should nevertheless be better governed by a variable number of active flavors.
\end{abstract}


The fixed flavor factorization schme (FFS) is characterized by considering the heavy quarks $(h=c, b, t)$ always as external particles which are not included among the partons in the colorless hadrons. Their participation in deep inelastic scattering processes like, say, $e N \rightarrow e X$ is considered to be due to production subprocesses such as $\gamma^{*} g \rightarrow h \bar{h}$ rather than $\gamma^{*} h \rightarrow h$. The latter subprocess becomes relevant in the so called variable flavor factorization scheme where, besides the light $u, d, s$ quarks, the heavy quarks are also considered to form an intrinsic part of colorless hadrons. Considering both subprocesses together would amount to double counting and thus the FFS dictates setting the number of flavors $n_{f}=3$ in the flavor-singlet QCD evolution equations

$$
\frac{d}{d \ln Q^{2}} \vec{q}\left(x, Q^{2}\right)=\frac{\alpha_{s}\left(Q^{2}\right)}{2 \pi} \int_{x}^{1} \frac{d y}{y} \hat{P}\left(\frac{x}{y}, Q^{2}\right) \vec{q}\left(y, Q^{2}\right)
$$

where $\vec{q}=(\Sigma, g)^{T}$ with

$$
\Sigma\left(x, Q^{2}\right)=\sum_{q=u, d, s}(q+\bar{q})
$$

and $\hat{P}\left(z, Q^{2}\right)=\hat{P}^{(0)}(z)+\frac{\alpha_{s}}{2 \pi} \hat{P}^{(1)}(z)+\left(\frac{\alpha_{s}}{2 \pi}\right)^{2} \hat{P}^{(2)}(z)$. In order to keep our arguments transparent as far as possible, we neglect, without loss of generality, the NLO (2-loop) and NNLO (3-loop) splitting functions $\hat{P}^{(1)}$ and $\hat{P}^{(2)}$, respectively, and the well known leading order (LO) splitting functions are given by [1]

$$
\hat{P}^{(0)}=\left(\begin{array}{cc}
P_{q q}^{(0)}, & 2 n_{f} P_{q g}^{(0)} \\
P_{g q}^{(0)}, & P_{g g}^{(0)}
\end{array}\right)
$$

with $P_{q q}^{(0)}(z)=C_{F}\left(\frac{1+z^{2}}{1-z}\right)_{+}, P_{q g}^{(0)}(z)=T_{R}\left[z^{2}+(1-z)^{2}\right], P_{g q}^{(0)}(z)=C_{F}\left[1+(1-z)^{2}\right] / z$ and

$$
P_{g g}^{(0)}(z)=2 C_{A}\left[\frac{z}{(1-z)}+\frac{1-z}{z}+z(1-z)\right]+c_{\delta} \delta(1-z)
$$

where $C_{F}=\frac{4}{3}, C_{A}=3$ and $T_{R}=\frac{1}{2}$. The $n_{f}$ dependence of $P_{g g}^{(0)}(z)$ resides in the endpoint $(z=1)$ contribution $c_{\delta}=\frac{11}{6} C_{A}-\frac{2 n_{f}}{3} T_{R}$ which derives from the energy-momentum sum rule

$$
\int_{0}^{1}\left[x \Sigma\left(x, Q^{2}\right)+x g\left(x, Q^{2}\right)\right] d x=1
$$


dictating the 'second moment' constraints

$$
\begin{gathered}
\int_{0}^{1} z\left[P_{q q}^{(0)}(z)+P_{g q}^{(0)}(z)\right] d z=0 \\
\int_{0}^{1} z\left[2 n_{f} P_{q g}^{(0)}(z)+P_{g g}^{(0)}(z)\right] d z=0 .
\end{gathered}
$$

Inserting (4) into this latter constraint gives $2 n_{f} T_{R} \frac{1}{3}+\left(-\frac{11}{6} C_{A}+c_{\delta}\right)=0$, i.e. $c_{\delta}$ given above. In other words, the $n_{f}$ dependence of $P_{g g}^{(0)}$ derives, as is well known [1], from the 'second moment' of the splitting function $2 n_{f} P_{q g}^{(0)}$ describing the splitting of the gluon $g \rightarrow q \bar{q}$ into $n_{f}$ massless on-shell quark-antiquark pairs. The choice $n_{f}=3$ in (3) is dictated by the FFS expression for $\Sigma\left(x, Q^{2}\right)$ in (2). Consequently, fixing $n_{f}=3$ in $P_{g g}^{(0)}$ in (4) is needed to guarantee the $Q^{2}$ independence of the energy-momentum sum rule (5).

On the other hand, the running coupling $\alpha_{s}\left(Q^{2}\right)$ in (1) evolves according to

$$
\frac{d \alpha_{s}\left(Q^{2}\right)}{d \ln Q^{2}}=\beta\left(\alpha_{s}\right)=-\beta_{0} \frac{\alpha_{s}^{2}\left(Q^{2}\right)}{4 \pi}+\ldots
$$

where

$$
\beta_{0}=11-\frac{2}{3} n_{f}
$$

and $n_{f}$ is the number of active quark flavors $f$ satisfying, in the most commonly used $\overline{\mathrm{MS}}$ renormalization scheme, $m_{f}^{2} \leq Q^{2}$. As is well known, this $n_{f}$ dependence in (8) derives from the same fermionic 1-loop vacuum polarization diagram as in QED, by properly taking into account the color degrees of freedom. Furthermore, it is the scale (virtuality) $Q^{2}$ which dictates how many quark flavors effectively contribute in (7). The question arises whether the FFS also dictates [2] setting always $n_{f}=3$ in $\beta_{0}$. First of all one notes that this is not needed in order to guarantee the $Q^{2}$ independence of (5). Secondly the inclusion of the heavy quark $h$-loops in the vacuum polarization diagrams, responsible for the $n_{f}$ dependence in $\beta_{0}$, does not involve double counting since this contribution is not accounted for in the FFS as explained above. For this second reason one may keep the $n_{f}$ dependence in $\beta_{0}$, i.e. in $\alpha_{s}\left(Q^{2}\right)$, in contrast to the situation in (3) and (4) where 
it would lead to double counting in the FFS since here the heavy quark flavors $h=c, b, t$ are already taken care of via the production process $\gamma^{*} g \rightarrow h \bar{h}$ as argued above.

It is, of course, also possible to fix $n_{f}=3$ in the $\beta$-function governing the evolution of $\alpha_{s}\left(Q^{2}\right)$ as was considered in [2]. But then one encounters large higher order $\alpha_{s}^{\left(n_{f}=3\right)}\left(Q^{2}\right) \ln \frac{Q^{2}}{m_{h}^{2}} \operatorname{logarithmic}$ corrections in all subsequent calculations like, e.g., the $Q^{2}$ evolution equations for the $\mathrm{u}, \mathrm{d}, \mathrm{s}$ and gluon distributions, which must be taken into account. For this reason one should better choose for the calculation of these distributions the variable flavor number scheme in the $\beta$-function, even in the so called fixed flavor factorization scheme, a practice followed in many analyses carried out within this factorization scheme. Making this choice, automatically resums the above mentioned higher order logarithmic corrections and consequently improves the stability of the perturbative expansion. At any rate, simply fixing [2] $n_{f}=3$ in $\beta_{0}$ and $\beta_{1}$ does not generate the correct $Q^{2}$-evolution of the $\mathrm{u}, \mathrm{d}, \mathrm{s}$ and $g$ distributions. A possible objection to the variable flavor scheme in $\beta\left(\alpha_{s}\right)$ could be that the NLO coefficient functions for the $c \bar{c}$ pair production have been calculated [3] in a renormalization scheme where the coupling $\alpha_{s}$ evolves according to $n_{f}=3$. However, as demonstrated in [4, it is perfectly appropriate to choose $\mu_{r}=\mu_{f}=\mathcal{O}\left(m_{c}\right)$ for the renormalization and factorization scales in calculating the NLO $c \bar{c}$ pair production cross section, i.e., a scale $\mu_{r}$ where $n_{f}=3$ in $\beta\left(\alpha_{s}\right)$ is the appropriate choice.

Let us finally note that the argument in [2] for fixing $n_{f}=3$ in $\beta_{0}$ is actually not conclusive. This can be easily seen by considering the LO quark contribution to the longitudinal structure function $F_{L}$ which results in an $n_{f}$ dependence of $\partial F_{L} / \partial \ln Q^{2}$ in contrast to the situation presented in [2] where only the gluon contribution to $F_{L}$ was taken into account. Furthermore, it is well known that also $\partial F_{2} / \partial \ln Q^{2}$ is $n_{f}$ dependent. Thus the demand [2] for the $n_{f}$ independence of these quantities does not provide any meaningful criterion and can moreover never be realized. 
To summarize, in contrast to the assertions in [2], we conclude that it is neither wrong nor inconsistent to choose a scale dependent $n_{f}$ in $\beta\left(\alpha_{s}\right)$ within the fixed flavor factorization scheme. The arguments presented here, mainly within the LO framework, hold obviously also for the higher perturbative orders, but their explicit demonstration is technically more involved and consequently less transparent.

\section{References}

[1] G. Altarelli and G. Parisi, Nucl. Phys. B126 (1977) 298

[2] A.D. Martin, W.J. Stirling, and R.S. Thorne, Phys. Lett. B636 (2006) 259

[3] E. Laenen et al., Nucl. Phys. B392 (1993) 162;

B.W. Harris and J. Smith, Nucl. Phys. B452 (1995) 109;

P. Nason, S. Dawson, and R.K. Ellis, Nucl. Phys. B303 (1988) 607;

R.K. Ellis and P. Nason, Nucl. Phys. B312 (1989) 551.

[4] M. Glück, E. Reya, and M. Stratmann, Nucl. Phys. B422 (1994) 37 